\title{
Einstein-like Lorentzian Lie groups of dimension four
}

Amirhesam Zaeim

To cite this article: Amirhesam Zaeim (2017) Einstein-like Lorentzian Lie groups of dimension four, Journal of Nonlinear Mathematical Physics 24:4, 556-570, DOI: https://doi.org/10.1080/14029251.2017.1375691

To link to this article: https://doi.org/10.1080/14029251.2017.1375691

Published online: 04 January 2021 


\title{
Einstein-like Lorentzian Lie groups of dimension four
}

\author{
Amirhesam Zaeim \\ Department of Mathematics, Payame Noor University, P.O. Box 19395-3697 \\ Tehran, I.R. of Iran. \\ zaeim@pnu.ac.ir
}

Received 11 February 2017

Accepted 15 June 2017

\begin{abstract}
Einstein-like examples of four-dimensional Lorentzian Lie groups are listed and geometric properties of each class have been investigated.

Keywords: Lie groups; left-invariant metrics; Einstein like metrics.
\end{abstract}

2000 Mathematics Subject Classification: 53C50, 53C15, 53C25

\section{Introduction}

Einstein-like manifolds which are introduced by A. Gray [15], firstly defined as a generalization of Einstein property in two new classes of Riemannian manifolds. He called these two classes as $\mathscr{A}$ and $\mathscr{B}$, which are defined by special conditions on the Ricci tensor. These definitions could be immediately extended to the pseudo-Riemannian geometry and we bring them in here.

- The Ricci tensor $\rho$ of a pseudo-Riemannian manifold $(M, g)$ is cyclic-parallel, that is,

$$
\left(\nabla_{X} \rho\right)(Y, Z)+\left(\nabla_{Y} \rho\right)(Z, X)+\left(\nabla_{Z} \rho\right)(X, Y)=0, \quad \forall X, Y, Z \in \mathfrak{X}(M) .
$$

In this case we say, $(M, g)$ belongs to class $\mathscr{A}$. This condition is equivalent to requiring that $\rho$ is a Killing tensor, that is,

$$
\left(\nabla_{X} \rho\right)(X, X)=0
$$

The Equation (1.2), which is also known as the first odd Ledger condition, is a necessary condition for a (pseudo-)Riemannian manifold to be a D'Atri space. Hence, to study those spaces satisfying the Equation (1.2), is the first step to understand D'Atri spaces of a specific dimension.

- The Ricci tensor of a pseudo-Riemannian manifold $(M, g)$ is a Codazzi tensor, that is,

$$
\left(\nabla_{X} \rho\right)(Y, Z)=\left(\nabla_{Y} \rho\right)(X, Z), \quad \forall X, Y, Z \in \mathfrak{X}(M) .
$$

In this case we say, $(M, g)$ belongs to class $\mathscr{B}$. 
In the following diagram, we see the relation between different classes of manifolds.

$$
\begin{aligned}
& \mathscr{E} \subset \mathscr{P}=\mathscr{A} \cap \mathscr{B}{ }^{\subset \mathscr{A}} \subset \mathscr{A} \cup \mathscr{B} \subset \mathscr{C}, \\
& \subset \mathscr{B}
\end{aligned}
$$

where the class of Ricci parallel, Einstein and manifolds with constant scalar curvature are denoted by $\mathscr{P}, \mathscr{E}$ and $\mathscr{C}$ respectively.

Homogeneous spaces through Rimannian and pseudo-Riemnnian geometries, have been subject of several studies for the Einstein-like property. These researches show the importance of Einsteinlike metrics, particularly between homogeneous manifolds. In the Riemannian homogeneous spaces of dimension three, Einstein-like metrics were studied in [2]. The class of Einstein-like Riccicurvature homogeneous spaces was the subject of [7]. In this research, authors could completely classify the three-dimensional case, while the problem was solved for locally homogeneous metrics of dimension four. Einstein-like metrics were also studied between ball-homogeneous spaces of dimension three [8], semi-symmetric spaces [5], Sasakian spaces [1] and three-dimensional contact metric manifolds [9].

About the pseudo-Riemannian case, almost all of investigations are focused on the three dimensional Lorentzian manifolds. Einstein-like metrics of the three-dimensional homogeneous Lorentzian manifolds were completely classified in [10], while the same author studied Einsteinlike curvature homogeneous up to order one Lorentzian manifolds in [12]. He also classified three-dimensional curvature homogeneous Lorentzian manifolds equipped with either Einsteinlike or conformally flat metrics in [11]. Einstein-like metrics studied through the class of threedimensional Walker manifolds in [14]. A classification of four-dimensional pseudo-Riemannian homogeneous manifolds with non-trivial isotropy was given in [16]. According to this study, classification of Einstein-like homogeneous pseudo-Riemannian four manifolds with non-trivial isotropy was obtained in [21]. Finally, four-dimensional Lie groups equipped with a left-invariant Lorentzian metric, obtaining a complete classification of the Einstein and Ricci-parallel examples, were studied in [13]. Now, it is a natural question to investigate Einstein-like Lie groups with a left-invariant Lorentzian metric (briefly, Lorentzian Lie groups) in dimension four. The aim of the present paper is to explicitly find the Einstein-like examples of four-dimensional Lorentzian Lie groups.

This paper is organized as following. In the next section, the basic point of this study which is the general form of four-dimensional Lorentzian Lie groups is presented. Einstein-like classes $\mathscr{A}$ and $\mathscr{B}$ are investigated in the next two sections respectively and we present a complete list of possible examples with their geometric properties.

\section{Lorentzian Lie groups}

Four-dimensional Riemannian Lie groups classified in the well-known work of Bérard-Bérgery [4]. In particular, according to his classification results, he proved that a (simply connected) fourdimensional homogeneous Riemannian manifold is either symmetric, or isometric to a Lie group equipped with a left-invariant Riemannian metric. Explicitly, with regard to Riemannian Lie groups, one has the following (see for example [3]).

Proposition 2.1. The simply connected four-dimensional Riemannian Lie groups are:

(i) the direct products $\mathbb{R} \times S U(2)$ and $\mathbb{R} \times \widetilde{S L}(2, \mathbb{R})$ (unsolvable); 
(ii) one of the following solvable Lie groups:

(iil) the non-trivial semi-direct products $\mathbb{R} \ltimes E(2)$ and $\mathbb{R} \ltimes E(1,1)$;

(ii2) the non-nilpotent semi-direct products $\mathbb{R} \ltimes H$ (H denoting the Heisenberg group);

(ii3) the semi-direct products $\mathbb{R} \ltimes \mathbb{R}^{3}$.

The description of four-dimensional Lie groups equipped with a left-invariant Lorentzian metric shows that the class of Lorentzian Lie groups is the same as Riemannian ones. In fact the following proposition holds (see [13]).

Proposition 2.2. The class of n-dimensional (connected) Lorentzian Lie groups (respectively, Lorentzian Lie algebras) coincides with the class of the Riemannian ones. In particular, if $G$ is a four-dimensional simply connected Lorentzian Lie group, then it is one of Lie groups listed in Proposition 2.1.

Let the Lie algebra of $G$ be given by $\mathfrak{g}=\mathfrak{r} \ltimes \mathfrak{g}_{3}$, that is, $\mathfrak{g}=\mathfrak{r} \oplus \mathfrak{g}_{3}$, where $\mathfrak{g}_{3}$ is a threedimensional Lie algebra, and the generator of the one-dimensional Lie algebra $\mathfrak{r}$ acts as a derivation on $\mathfrak{g}_{3}$. Considering the geometric properties of Lorentzian inner products over a four-dimensional Lie algebra $\mathfrak{g}$, we have a fundamental difference with respect to the Riemannian case. In fact, let $g$ denote an inner product over $\mathfrak{g}=\mathfrak{r} \ltimes \mathfrak{g}_{3}$. If $g$ is Riemannian, then so is its restriction over $\mathfrak{g}_{3}$. And three-dimensional Riemannian Lie groups are well known since the seminal work [17]. But in the Lorentzian case the restriction of $g$ over $\mathfrak{g}_{3}$ is

(a) either positive definite, (b) Lorentzian, or (c) degenerate.

This difference is the key point in the study of the Lorentzian Lie groups as the authors considered in [13]. We report the following proposition.

Proposition 2.3. [13] Let $(\mathfrak{g}, g)$ be an arbitrary four-dimensional Lorentzian Lie algebra. Then, there exists a basis $\left\{e_{1}, e_{2}, e_{3}, e_{4}\right\}$ of $\mathfrak{g}$, such that

- $\mathfrak{g}_{3}=\operatorname{Span}\left(e_{1}, e_{2}, e_{3}\right)$ is a three-dimensional Lie algebra and $e_{4}$ acts as a derivation on $\mathfrak{g}_{3}$ (that is, $\mathfrak{g}=\mathfrak{r} \ltimes \mathfrak{g}_{3}$, where $\mathfrak{r}=\operatorname{Span}\left(e_{4}\right)$ ), and

- with respect to $\left\{e_{1}, e_{2}, e_{3}, e_{4}\right\}$, the Lorentzian inner product takes one of the following forms:

$$
\text { (a) }\left(\begin{array}{cccc}
1 & 0 & 0 & 0 \\
0 & 1 & 0 & 0 \\
0 & 0 & -1 & 0 \\
0 & 0 & 0 & 1
\end{array}\right), \quad(b)\left(\begin{array}{cccc}
1 & 0 & 0 & 0 \\
0 & 1 & 0 & 0 \\
0 & 0 & 1 & 0 \\
0 & 0 & 0 & -1
\end{array}\right), \quad(c)\left(\begin{array}{cccc}
1 & 0 & 0 & 0 \\
0 & 1 & 0 & 0 \\
0 & 0 & 0 & 1 \\
0 & 0 & 1 & 0
\end{array}\right) .
$$

The proposition 2.3, permits to study the geometric properties of Lorentzian Lie algebras (specially Einstein-like property). We investigate this matter in the next sections.

\section{Examples of class $\mathscr{A}$}

The Ricci parallel examples of Lorentzian Lie groups are completely listed and geometrically considered in [13]. For this reason, we exclude the Ricci parallel examples from the list of Einstein-like Lorentzain Lie groups. The cyclic parallel examples, i.e. those of class $\mathscr{A}$, are studied through the following theorem.

Theorem 3.1. Let $G$ be a four-dimensional simply connected Lie group equipped with a left invariant Lorentzian metric g. If $(G, g)$ belongs to class $\mathscr{A}$ (and not Ricci parallel), then the Lie algebra 
$\mathfrak{g}$ of $G$ is isometric to $\mathfrak{g}=\mathfrak{r} \ltimes \mathfrak{g}_{3}$, where $\mathfrak{g}_{3}=\operatorname{span}\left\{e_{1}, e_{2}, e_{3}\right\}$ and $\mathfrak{r}=\operatorname{span}\left\{e_{4}\right\}$, and one of the following cases occurs.

(a) $\left\{e_{i}\right\}_{i=1}^{4}$ is a pseudo-orthonormal basis with $e_{3}$ time-like. In this case, $G$ is isometric to one of the following semi-direct products $\mathbb{R} \ltimes G_{3}$.

a1) $\mathbb{R} \ltimes H$, where $H$ is the Heisenberg group and $\mathfrak{g}$ is described by one of the following sets of conditions:

1) $\left[e_{1}, e_{3}\right]=A e_{2}+B e_{3},\left[e_{1}, e_{4}\right]=\frac{A C}{B} e_{2}+C e_{3},\left[e_{3}, e_{4}\right]=\frac{A D}{B} e_{2}+D e_{3}$, with $A^{2}+C^{2} \neq 0, A \neq \pm B$,

2) $\left[e_{1}, e_{3}\right]=A e_{1}+B e_{2}+C e_{3}$, with $B \neq 0, A^{2}+B^{2}-C^{2} \neq 0$,

3) $\left[e_{1}, e_{3}\right]=A e_{1}+B e_{3},\left[e_{1}, e_{4}\right]=\frac{A C}{B} e_{1}+C e_{3},\left[e_{3}, e_{4}\right]=\frac{A D}{B} e_{1}+D e_{3}$, with $C^{2}+D^{2} \neq 0, A \neq \pm B, A D+C B \neq 0$,

4) $\left[e_{1}, e_{3}\right]=A e_{1}+\frac{C D}{B} e_{2}+C e_{3},\left[e_{1}, e_{4}\right]=\frac{-A^{2} B}{C^{2}} e_{1}-\frac{A D}{C} e_{2}-\frac{A B}{C} e_{3},\left[e_{3}, e_{4}\right]=\frac{A B}{C} e_{1}+D e_{2}+B e_{3}$, with $D \neq 0, A B \pm C \sqrt{B^{2}-D^{2}} \neq 0, A B \pm C \sqrt{B^{2}+C^{2}} \neq 0$,

5) $\left[e_{1}, e_{2}\right]=A e_{2},\left[e_{1}, e_{3}\right]=B e_{2},\left[e_{1}, e_{4}\right]=C e_{2},\left[e_{2}, e_{3}\right]=D e_{2},\left[e_{2}, e_{4}\right]=E e_{2},\left[e_{3}, e_{4}\right]=$ $\frac{E B-D C}{A} e_{2}$

with $B^{2}+C^{2} \neq 0, B^{2}+E^{2}+(A \pm D)^{2} \neq 0$,

$B\left(\left(A B \pm E \sqrt{C^{2}-B^{2}}\right)^{2}+(B D-C E)^{2}\right) \neq 0$,

6) $\left[e_{2}, e_{3}\right]=A e_{2},\left[e_{2}, e_{4}\right]=B e_{2},\left[e_{3}, e_{4}\right]=C e_{2}$, with $A C \neq 0$,

a2) $\mathbb{R} \ltimes E(2)$ or $\mathbb{R} \ltimes E(1,1)$, with $\mathfrak{g}$ is described by one of the following sets of conditions:

7) $\left[e_{1}, e_{2}\right]=-\frac{\varepsilon}{2} \sqrt{-4 A^{2}+2 B^{2}} e_{2},\left[e_{1}, e_{3}\right]=\frac{\delta 3}{2} \sqrt{-2 A^{2}+B^{2}} e_{2}+\frac{\varepsilon}{2} \sqrt{-4 A^{2}+2 B^{2}} e_{3},\left[e_{1}, e_{4}\right]=$ $\frac{\varepsilon \delta \sqrt{2}}{2} B e_{2}+B e_{3},\left[e_{2}, e_{4}\right]=-A e_{2},\left[e_{3}, e_{4}\right]=\frac{\varepsilon \delta 3 \sqrt{2}}{2} A e_{2}+A e_{3}$, with $B \neq 0, B^{2} \neq 2 A^{2}$,

8) $\left[e_{1}, e_{2}\right]=\frac{A}{2} e_{2},\left[e_{1}, e_{3}\right]=\frac{\varepsilon A(2 B-C)}{\sqrt{A^{2}+C^{2}}} e_{1}+A e_{3},\left[e_{1}, e_{4}\right]=(2 B-C) e_{1}+\varepsilon \sqrt{C^{2}+A^{2}} e_{3},\left[e_{2}, e_{3}\right]=$ $\frac{\varepsilon A(2 B-C)}{2 \sqrt{A^{2}+C^{2}}} e_{2},\left[e_{2}, e_{4}\right]=B e_{2},\left[e_{3}, e_{4}\right]=\frac{\varepsilon C(2 B-C)}{\sqrt{A^{2}+C^{2}}} e_{1}+C e_{3}$,

with $A^{2}+B^{2} \neq 0, A^{2}+(B-C)^{2} \neq 0, C^{2}+(A \pm 2 B)^{2} \neq 0, A^{2}+2 C B \neq 0, B \neq \frac{1}{2}(C \pm$ $\left.\sqrt{A^{2}+C^{2}}\right)$.

a3) $\mathbb{R} \ltimes \mathbb{R}^{3}$ with $\mathfrak{g}$ is described by one of the following sets of conditions:

9) $\left[e_{1}, e_{4}\right]=\frac{B C}{A} e_{2}+B e_{3},\left[e_{3}, e_{4}\right]=C e_{2}+A e_{3}$,

with $B^{2}+C^{2} \neq 0, C \neq \pm A$,

10) $\left[e_{1}, e_{4}\right]=A e_{2},\left[e_{2}, e_{4}\right]=B e_{2},\left[e_{3}, e_{4}\right]=C e_{2}$,

with $A^{2}+C^{2} \neq 0,(A \pm C)^{2}+B^{2} \neq 0$,

11) $\left[e_{1}, e_{4}\right]=A e_{2}+B e_{3}$, with $A \neq \pm B$,

12) $\left[e_{2}, e_{4}\right]=A e_{1}+B e_{2}$, with $A \neq 0$,

13) $\left[e_{1}, e_{4}\right]=\varepsilon A e_{2}+\delta \sqrt{2} A e_{3},\left[e_{2}, e_{4}\right]=-A e_{2},\left[e_{3}, e_{4}\right]=\frac{\varepsilon \delta 3 \sqrt{2}}{2} A e_{2}+A e_{3}$, with $A \neq 0$,

14) $\left[e_{1}, e_{4}\right]=A e_{1}+B e_{2},\left[e_{2}, e_{4}\right]=\frac{A C}{B} e_{1}+C e_{2}$, with $A C \neq B^{2}$, 
(b) $\left\{e_{i}\right\}_{i=1}^{4}$ is a pseudo-orthonormal basis with $e_{4}$ time-like. In this case, $G$ is isometric to one of the following semi-direct products $\mathbb{R} \ltimes G_{3}$.

b1) $\mathbb{R} \ltimes H$, where $H$ is the Heisenberg group and $\mathfrak{g}$ is described by one of the following sets of conditions:

15) $\left[e_{1}, e_{3}\right]=A e_{1}+B e_{2}+C e_{3}$, with $B \neq 0$,

16) $\left[e_{1}, e_{3}\right]=A e_{1}+\frac{C D}{B} e_{2}+C e_{3},\left[e_{1}, e_{4}\right]=\frac{A^{2} B}{C^{2}} e_{1}+\frac{A D}{C} e_{2}+\frac{A B}{C} e_{3},\left[e_{3}, e_{4}\right]=\frac{A B}{C} e_{1}+D e_{2}+$ $B e_{3}$, with $D \neq 0, A \neq \frac{ \pm C \sqrt{C^{2}-B^{2}}}{B}$,

b2) $\mathbb{R} \ltimes E(2)$ or $\mathbb{R} \ltimes E(1,1)$, with $\mathfrak{g}$ is described by one of the following sets of conditions:

17) $\left[e_{1}, e_{2}\right]=\frac{A}{2} e_{2},\left[e_{1}, e_{3}\right]=-\frac{\varepsilon A(B-2 C)}{\sqrt{A^{2}-B^{2}}} e_{1}+A e_{3},\left[e_{1}, e_{4}\right]=(2 C-B) e_{1}+\varepsilon \sqrt{A^{2}-B^{2}} e_{3},\left[e_{2}, e_{3}\right]=$ $-\frac{\varepsilon A(B-2 C)}{2 \sqrt{A^{2}-B^{2}}} e_{2},\left[e_{2}, e_{4}\right]=C e_{2},\left[e_{3}, e_{4}\right]=-\frac{\varepsilon B(B-2 C)}{\sqrt{A^{2}-B^{2}}} e_{1}+B e_{3}$, with $A^{2}+(B-C)^{2} \neq 0, A^{2}-2 B C \neq 0, C \neq \frac{1}{2}\left(B \pm \sqrt{B^{2}-A^{2}}\right)$.

18) $\left[e_{1}, e_{2}\right]=\frac{\varepsilon A}{2} e_{2},\left[e_{1}, e_{3}\right]=B e_{1}+\varepsilon A e_{3},\left[e_{2}, e_{3}\right]=\frac{B}{2} e_{2},\left[e_{2}, e_{4}\right]=\frac{A}{2} e_{2},\left[e_{3}, e_{4}\right]=\varepsilon B e_{1}+$ $A e_{3}$, with $B \neq 0$,

b3) $\mathbb{R} \ltimes \mathbb{R}^{3}$ with $\mathfrak{g}$ is described by one of the following sets of conditions:

19) $\left[e_{1}, e_{4}\right]=\frac{\varepsilon \sqrt{2} A}{2} e_{2}+A e_{3},\left[e_{2}, e_{4}\right]=-\frac{3 \varepsilon \sqrt{2} A}{4} e_{1}$, with $A \neq 0$,

(c) $\left\{e_{i}\right\}_{i=1}^{4}$ is a basis with the inner product completely determined by $g\left(e_{1}, e_{1}\right)=g\left(e_{2}, e_{2}\right)=$ $g\left(e_{3}, e_{4}\right)=g(e 4, e 3)=1$ and $g\left(e_{i}, e_{j}\right)=0$ otherwise. In this case, $G$ is isometric to one of the following semi-direct products $\mathbb{R} \ltimes G_{3}$.

c1) $\mathbb{R} \ltimes H$, where $H$ is the Heisenberg group and $\mathfrak{g}$ is described by one of the following sets of conditions:

20) $\left[e_{1}, e_{2}\right]=\frac{B C}{A} e_{1}+B e_{2}+\frac{B D}{A} e_{3},\left[e_{1}, e_{4}\right]=-\frac{E B C}{A^{2}} e_{1}-\frac{B E}{A} e_{2}-\frac{B D E}{A^{2}} e_{3},\left[e_{2}, e_{3}\right]=C e_{1}+A e_{2}+$ $D e_{3},\left[e_{2}, e_{4}\right]=\frac{C F}{A} e_{1}+F e_{2}+\frac{F D}{A} e_{3},\left[e_{3}, e_{4}\right]=\frac{E C}{A} e_{1}+E e_{2}+\frac{E D}{A} e_{3}, F^{2}+(B+\varepsilon E)^{2}+(A D+$ $E(A+\varepsilon C))^{2} \neq 0,(B+\varepsilon \sqrt{3} E)^{2}+(A D+E(A+\varepsilon \sqrt{3} C))^{2}+\left(A F+E^{2}\right)^{2} \neq 0, C^{2}+(D+E)^{2} \neq$ $0,\left(A+\frac{B^{2}-E^{2}}{2 F}\right)^{2}+\left(D+\frac{-E^{3}+E B^{2}+2 C B F}{B^{2}-E^{2}}\right)^{2} \neq 0,\left(B+\varepsilon \frac{\sqrt{3}}{3} E\right)^{2}+(3 A D+E(3 A+\varepsilon \sqrt{3} C))^{2}+$ $\left(3 A F-E^{2}\right)^{2} \neq 0$,

21) $\left[e_{1}, e_{2}\right]=A e_{1}+B e_{2}+C e_{3},\left[e_{1}, e_{4}\right]=D e_{1}+\frac{D B}{A} e_{2}+\frac{C D}{A} e_{3}, \quad\left[e_{2}, e_{4}\right]=E e_{1}+\frac{B E}{A} e_{2}+$ $\frac{C E}{A} e_{3},\left(B-\frac{A(E+C)}{D}\right)^{2} \neq 0, D^{2}+(E+C)^{2} \neq 0$,

c2) $\mathbb{R} \ltimes E(2)$ or $\mathbb{R} \ltimes E(1,1)$, with $\mathfrak{g}$ is described by one of the following sets of conditions:

22) $\left[e_{1}, e_{2}\right]=A e_{1}-B e_{2}+\frac{2 A^{2}+B^{2}}{2 C} e_{3}, \quad\left[e_{1}, e_{3}\right]=-2 C e_{2}+$ $B e_{3},\left[e_{1}, e_{4}\right]=-\frac{A D}{C} e_{1}-\frac{-3 B^{2}+8 A^{2}-4 D^{2}+4 D B}{8 C} e_{2}+\frac{-3 B^{3}+8 A^{2} B-4 B D^{2}+4 D B^{2}-16 D A^{2}}{16 C^{2}} e_{3}, \quad\left[e_{2}, e_{3}\right]=$ $C e_{1}+A e_{3},\left[e_{2}, e_{4}\right]=-\frac{-12 D B+3 B^{2}+8 A^{2}+4 D^{2}}{16 C} e_{1}+\frac{A B}{C} e_{2}-\frac{A\left(11 B^{2}+8 A^{2}+4 D^{2}-12 D B\right)}{16 C^{2}} e_{3},\left[e_{3}, e_{4}\right]=$ $D e_{1}+2 A e_{2}-\frac{A(B-D)}{C} e_{3}, B-2 D \neq 0$,

c3) $\mathbb{R} \ltimes \widetilde{S L}(2, \mathbb{R})$ or $\mathbb{R} \ltimes S U(2)$ with $\mathfrak{g}$ is described by one of the following sets of conditions: 
23) $\left[e_{1}, e_{2}\right]=A e_{1}-B e_{2}-\frac{-2 D A+B^{2}+A^{2}+2 D^{2}}{C} e_{3}, \quad\left[e_{1}, e_{3}\right]=C e_{2}+B e_{3}, \quad\left[e_{1}, e_{4}\right]=\frac{A B}{C} e_{1}-$ $\frac{-D^{2}+B^{2}}{2 C} e_{2}-\frac{B\left(3 D^{2}+B^{2}-4 D A+2 A^{2}\right)}{2 C^{2}} e_{3},\left[e_{2}, e_{3}\right]=-2 C e_{1}+A e_{3},\left[e_{2}, e_{4}\right]=\frac{-B^{2}-D^{2}+D A}{C} e_{1}-\frac{D B}{C} e_{2}-$ $\frac{-5 A D i^{2}-B^{2} A+2 D B^{2}+2 D A^{2}+4 D^{3}}{2 C^{2}} e_{3},\left[e_{3}, e_{4}\right]=2 B e_{1}+D e_{2}-\frac{B(-D+A)}{C} e_{3}, A-2 D \neq 0$,

24) $\left[e_{1}, e_{2}\right]=A e_{1}-B e_{2}+\frac{2 D^{2}-2 D B+B^{2}+A^{2}}{C} e_{3}, \quad\left[e_{1}, e_{3}\right]=-2 C e_{2}+B e_{3}, \quad\left[e_{1}, e_{4}\right]=-\frac{A D}{C} e_{1}-$ $\frac{A^{2}+D^{2}-D B}{C} e_{2}+\frac{A^{2} B+5 B D^{2}-2 D B^{2}-4 D^{3}-2 D A^{2}}{2 C^{2}} e_{3},\left[e_{2}, e_{3}\right]=C e_{1}+A e_{3},\left[e_{2}, e_{4}\right]=-\frac{A^{2}-D^{2}}{2 C} e_{1}+$ $\frac{A B}{C} e_{2}-\frac{A\left(A^{2}+3 D^{2}-4 D B+2 B^{2}\right)}{2 C^{2}} e_{3},\left[e_{3}, e_{4}\right]=D e_{1}+2 A e_{2}-\frac{A(B-D)}{C} e_{3}, B-2 D \neq 0$,

25) $\left[e_{1}, e_{2}\right]=A e_{1}+B e_{3},\left[e_{1}, e_{3}\right]=-C e_{2}, \quad\left[e_{1}, e_{4}\right]=D e_{2},\left[e_{2}, e_{3}\right]=C e_{1}+A e_{3}, \quad\left[e_{2}, e_{4}\right]=$ $-\frac{C D+A^{2}}{C} e_{1}-\frac{A(B+D)}{C} e_{3},\left[e_{3}, e_{4}\right]=A e_{2}, B C-A^{2} \neq 0$,

26)

$$
\begin{aligned}
& {\left[e_{1}, e_{2}\right] \quad=\quad A e_{1}-\varepsilon A e_{2}+B e_{3}, \quad\left[e_{1}, e_{3}\right]=} \\
& -C e_{2}+\varepsilon A e_{3},\left[e_{1}, e_{4}\right]=-\frac{\varepsilon A^{2}}{C} e_{1}+D e_{2}+\frac{\varepsilon A\left(-C D+A^{2}-B C\right)}{C^{2}} e_{3},\left[e_{2}, e_{3}\right]=C e_{1}+A e_{3},\left[e_{2}, e_{4}\right]= \\
& -D e_{1}+\frac{\varepsilon A^{2}}{C} e_{2}+\frac{A\left(-C D+A^{2}-B C\right)}{C^{2}} e_{3},\left[e_{3}, e_{4}\right]=\varepsilon A e_{1}+A e_{2}, B C-2 A^{2} \neq 0,
\end{aligned}
$$

c4) $\mathbb{R} \ltimes \mathbb{R}^{3}$ with $\mathfrak{g}$ is described by one of the following sets of conditions:

27) $\left[e_{1}, e_{4}\right]=A e_{1}+\frac{C A}{B} e_{2}+\frac{A D}{B} e_{3},\left[e_{2}, e_{4}\right]=E e_{1}+\frac{E C}{B} e_{2}+\frac{D E}{B} e_{3},\left[e_{3}, e_{4}\right]=B e_{1}+C e_{2}+D e_{3}$,

Proof. Let $(G, g)$ be a four-dimensional Lorentzian Lie group. By Proposition 2.3, the Lie algebra $\mathfrak{g}$ of $G$ is a semi-direct product $\mathfrak{r} \ltimes \mathfrak{g}_{3}$, where $\mathfrak{r}=\operatorname{span}\left(e_{4}\right)$ acts on $\mathfrak{g}_{3}=\operatorname{span}\left(e_{1}, e_{2}, e_{3}\right)$, and the Lorentzian inner product on $\mathfrak{g}$ is described by one of the conditions (a),(b),(c). The general form of the semi-direct product Lie algebra $\mathfrak{g}=\mathfrak{r} \ltimes \mathfrak{g}_{3}$ is given by

$$
\begin{aligned}
& {\left[e_{1}, e_{2}\right]=a_{1} e_{1}+a_{2} e_{2}+a_{3} e_{3},\left[e_{1}, e_{3}\right]=b_{1} e_{1}+b_{2} e_{2}+b_{3} e_{3},} \\
& {\left[e_{1}, e_{4}\right]=c_{1} e_{1}+c_{2} e_{2}+c_{3} e_{3},\left[e_{2}, e_{3}\right]=d_{1} e_{1}+d_{2} e_{2}+d_{3} e_{3},} \\
& {\left[e_{2}, e_{4}\right]=p_{1} e_{1}+p_{2} e_{2}+p_{3} e_{3},\left[e_{3}, e_{4}\right]=q_{1} e_{1}+q_{2} e_{2}+q_{3} e_{3},}
\end{aligned}
$$

for some real constants $a_{i}, \ldots, q_{i}$, which, by virtue of the Jacobi identity, must satisfy

$$
\begin{aligned}
& a_{2} d_{1}+b_{3} d_{1}-b_{1} d_{3}-a_{1} d_{2}=0 \\
& a_{2} d_{3}+a_{1} b_{3}-b_{1} a_{3}-d_{2} a_{3}=0 \\
& d_{2} b_{3}-b_{1} a_{2}+a_{1} b_{2}-d_{3} b_{2}=0 \\
& b_{1} p_{3}-c_{3} d_{1}-q_{1} a_{3}+a_{1} p_{2}-a_{2} p_{1}=0, \\
& b_{1} q_{3}-b_{3} q_{1}+a_{1} q_{2}+d_{1} c_{2}-b_{2} p_{1}=0 \\
& b_{2} p_{1}-d_{3} q_{2}-d_{1} c_{2}+d_{2} q_{3}-a_{2} q_{1}=0 \\
& b_{2} p_{3}+b_{1} c_{3}-d_{3} c_{2}-c_{1} b_{3}-a_{3} q_{2}=0 \\
& d_{2} c_{3}-c_{1} a_{2}+a_{3} q_{2}+a_{1} c_{2}-b_{2} p_{3}=0, \\
& p_{2} d_{3}+b_{3} p_{1}-d_{2} p_{3}-c_{3} d_{1}-q_{1} a_{3}=0 \\
& b_{3} q_{2}+p_{2} b_{2}+b_{1} c_{2}-d_{2} c_{2}-c_{1} b_{2}-q_{3} b_{2}-a_{2} q_{2}=0 \\
& d_{3} c_{3}-c_{1} a_{3}+a_{3} q_{3}+a_{2} p_{3}+a_{1} c_{3}-b_{3} p_{3}-p_{2} a_{3}=0, \\
& q_{3} d_{1}-d_{3} q_{1}+p_{2} d_{1}-d_{2} p_{1}-a_{1} q_{1}+b_{1} p_{1}-c_{1} d_{1}=0 .
\end{aligned}
$$

We treat separately the cases (a),(b),(c) for the Lorentzian inner product $g$ on $\mathfrak{g}$.

Case (a). By means of the previous description of the Lie algebra, the Levi-Civita connection $\nabla$ of $\mathfrak{g}$ will be determined using the well known Koszul formula. We set $\Lambda_{i}=\nabla_{e_{i}}$ and with respect to 
the pseudo-orthonormal basis $\left\{e_{1}, e_{2}, e_{3}, e_{4}\right\}$, with $e_{3}$ time-like, we have:

$$
\begin{aligned}
& \Lambda_{1}=\left(\begin{array}{cccc}
0 & a_{1} & b_{1} & c_{1} \\
-a_{1} & 0 & \frac{b_{2}+d_{1}+a_{3}}{2} & \frac{c_{2}+p_{1}}{2} \\
b_{1} & \frac{b_{2}+d_{1}+a_{3}}{2} & 0 & \frac{-q_{1}+c_{3}}{2} \\
-c_{1} & \frac{-p_{1}-c_{2}}{2} & \frac{-q_{1}+c_{3}}{2} & 0
\end{array}\right), \Lambda_{2}=\left(\begin{array}{cccc}
0 & a_{2} & \frac{d_{1}+b_{2}-a_{3}}{2} & \frac{c_{2}+p_{1}}{2} \\
-a_{2} & 0 & d_{2} & p_{2} \\
\frac{d_{1}+b_{2}-a_{3}}{2} & d_{2} & 0 & \frac{-q_{2}+p_{3}}{2} \\
\frac{-p_{1}-c_{2}}{2} & -p_{2} & \frac{-q_{2}+p_{3}}{2} & 0
\end{array}\right), \\
& \Lambda_{3}=\left(\begin{array}{cccc}
0 & \frac{-d_{1}-a_{3}+b_{2}}{2} & -b_{3} & \frac{-c_{3}+q_{1}}{2} \\
\frac{-b_{2}+a_{3}+d_{1}}{2} & 0 & -d_{3} & \frac{-p_{3}+q_{2}}{2} \\
\frac{-b_{3}}{2} & -d_{3} & 0 & q_{3} \\
\frac{-q_{1}+c_{3}}{2} & \frac{-q_{2}+p_{3}}{2} & q_{3} & 0
\end{array}\right), \Lambda_{4}=\left(\begin{array}{cccc}
0 & \frac{-p_{1}+c_{2}}{2} & \frac{-q_{1}-c_{3}}{2} & 0 \\
\frac{-c_{2}+p_{1}}{2} & 0 & \frac{-q_{2}-p_{3}}{2} & 0 \\
\frac{-q_{1}-c_{3}}{2} & \frac{-q_{2}-p_{3}}{2} & 0 & 0 \\
0 & 0 & 0 & 0
\end{array}\right) .
\end{aligned}
$$

We now describe the curvature tensor, calculating $R\left(e_{i}, e_{j}\right)=\Lambda_{i} \Lambda_{j}-\Lambda_{j} \Lambda_{i}-\Lambda_{\left[e_{i}, e_{j}\right]}$ for all indices $i, j$, and deducing the curvature components $R_{i j k h}=g\left(R\left(e_{i}, e_{j}\right) e_{k}, e_{h}\right)$. We find

$$
\begin{aligned}
& \begin{aligned}
R_{1212}= & \frac{1}{2}\left(c_{2} p_{1}+d_{1} a_{3}-b_{2} a_{3}-b_{2} d_{1}\right)-\frac{1}{4}\left(b_{2}^{2}+d_{1}^{2}-c_{2}^{2}-p_{1}^{2}\right)+\frac{3}{4} a_{3}^{2}+b_{1} d_{2}-c_{1} p_{2}- \\
& a_{1}^{2}-a_{2}^{2},
\end{aligned} \\
& R_{1213}=\frac{1}{2}\left(c_{1} q_{2}-c_{1} p_{3}\right)-\frac{1}{4}\left(c_{2} q_{1}-c_{2} c_{3}+p_{1} q_{1}-p_{1} c_{3}\right)-a_{1} d_{2}+a_{2} b_{2}+a_{2} d_{1}+a_{1} b_{1}- \\
& a_{3} b_{3} \text {, } \\
& R_{1214}=\frac{1}{2}\left(b_{1} p_{3}-b_{1} q_{2}\right)+\frac{1}{4}\left(q_{1} d_{1}+q_{1} b_{2}-c_{3} d_{1}-c_{3} b_{2}\right)-\frac{3}{4}\left(q_{1} a_{3}-c_{3} a_{3}\right)+a_{1} p_{2}- \\
& a_{2} c_{2}-a_{2} p_{1}-a_{1} c_{1} \text {, } \\
& R_{1223}=\frac{1}{2}\left(p_{2} c_{3}-p_{2} q_{1}\right)+\frac{1}{4}\left(c_{2} q_{2}-c_{2} p_{3}+p_{1} q_{2}-p_{1} p_{3}\right)+a_{1} d_{1}+a_{1} b_{2}-b_{1} a_{2}+a_{2} d_{2}- \\
& a_{3} d_{3} \text {, } \\
& R_{1224}=\frac{1}{2}\left(d_{2} q_{1}-d_{2} c_{3}\right)-\frac{1}{4}\left(b_{2} q_{2}-b_{2} p_{3}+d_{1} q_{2}-d_{1} p_{3}\right)-\frac{3}{4}\left(a_{3} q_{2}-a_{3} p_{3}\right)-a_{1} c_{2}- \\
& a_{1} p_{1}+c_{1} a_{2}-a_{2} p_{2} \text {, } \\
& R_{1234}=\frac{1}{2}\left(b_{1} c_{2}+b_{1} p_{1}+p_{2} b_{2}+p_{2} d_{1}+p_{2} a_{3}-c_{1} d_{1}-c_{1} b_{2}+c_{1} a_{3}-d_{2} c_{2}-d_{2} p_{1}+\right. \\
& \left.a_{1} q_{1}-a_{1} c_{3}+a_{2} q_{2}-a_{2} p_{3}\right)-a_{3} q_{3} \text {. } \\
& \begin{aligned}
R_{1313}= & \frac{1}{2}\left(c_{3} q_{1}+b_{2} d_{1}-d_{1} a_{3}-b_{2} a_{3}\right)-\frac{1}{4}\left(d_{1}^{2}+a_{3}^{2}+c_{3}^{2}+q_{1}^{2}\right)+\frac{3}{4} b_{2}^{2}+a_{1} d_{3}-c_{1} q_{3}+b_{1}^{2}- \\
& b_{3}^{2},
\end{aligned} \\
& R_{1314}=\frac{1}{2}\left(a_{1} q_{2}-a_{1} p_{3}\right)+\frac{1}{4}\left(d_{1} c_{2}+d_{1} p_{1}+a_{3} c_{2}+a_{3} p_{1}\right)-\frac{3}{4}\left(b_{2} c_{2}+b_{2} p_{1}\right)+b_{1} q_{3}- \\
& b_{3} q_{1}+b_{3} c_{3}-b_{1} c_{1} \text {, } \\
& R_{1323}=-\frac{1}{2}\left(q_{3} c_{2}+q_{3} p_{1}\right)-\frac{1}{4}\left(q_{2} q_{1}-q_{2} c_{3}-p_{3} q_{1}+p_{3} c_{3}\right)-a_{1} b_{3}+b_{1} a_{3}+b_{1} d_{1}+b_{2} d_{2}- \\
& b_{3} d_{3} \text {, } \\
& R_{1324}=\frac{1}{2}\left(a_{1} c_{3}-a_{1} q_{1}+q_{3} b_{2}+q_{3} d_{1}+a_{3} q_{3}+c_{1} b_{2}-c_{1} a_{3}-c_{1} d_{1}-d_{3} q_{1}+d_{3} c_{3}-b_{1} c_{2}-\right. \\
& \left.b_{1} p_{1}-b_{3} q_{2}+b_{3} p_{3}\right)-p_{2} b_{2} \text {, } \\
& R_{1334}=\frac{1}{2}\left(d_{3} c_{2}+d_{3} p_{1}\right)+\frac{1}{4}\left(d_{1} q_{2}-d_{1} p_{3}+a_{3} q_{2}-a_{3} p_{3}\right)+\frac{3}{4}\left(b_{2} q_{2}-b_{2} p_{3}\right)-b_{1} c_{3}+ \\
& b_{1} q_{1}+c_{1} b_{3}-b_{3} q_{3} \text {. } \\
& R_{1414}=-\frac{1}{2}\left(c_{2} p_{1}+c_{3} q_{1}\right)+\frac{1}{4}\left(p_{1}^{2}-q_{1}^{2}\right)-\frac{3}{4}\left(c_{2}^{2}-c_{3}^{2}\right)-c_{1}^{2} \text {, } \\
& R_{1423}=\frac{1}{2}\left(b_{1} p_{1}+c_{1} b_{2}+c_{1} d_{1}+c_{1} a_{3}-a_{1} q_{1}-a_{1} c_{3}-b_{1} c_{2}\right)+d_{2} c_{2}-d_{3} c_{3}, \\
& R_{1424}=-\frac{1}{4}\left(q_{2} q_{1}+p_{3} q_{1}+q_{2} c_{3}\right)+\frac{3}{4} p_{3} c_{3}-c_{1} p_{1}-p_{2} c_{2}, \\
& R_{1434}=\frac{1}{4}\left(p_{1} q_{2}-c_{2} p_{3}+p_{1} p_{3}\right)+\frac{3}{4} c_{2} q_{2}+c_{1} q_{1}-c_{3} q_{3},
\end{aligned}
$$




$$
\begin{aligned}
R_{2323}= & \frac{1}{2}\left(b_{2} a_{3}+q_{2} p_{3}+d_{1} a_{3}+b_{2} d_{1}\right)-\frac{1}{4}\left(b_{2}^{2}+a_{3}^{2}+q_{2}^{2}+p_{3}^{2}\right)+\frac{3}{4} d_{1}^{2}-a_{2} b_{3}-p_{2} q_{3}+ \\
& d_{2}^{2}-d_{3}^{2}, \\
R_{2324}= & \frac{1}{2}\left(a_{2} c_{3}-a_{2} q_{1}\right)+\frac{1}{4}\left(b_{2} c_{2}+b_{2} p_{1}-a_{3} c_{2}-a_{3} p_{1}\right)-\frac{3}{4}\left(d_{1} c_{2}+d_{1} p_{1}\right)+d_{2} q_{3}- \\
& d_{3} q_{2}+d_{3} p_{3}-d_{2} p_{2}, \\
R_{2334}= & \frac{1}{2}\left(b_{3} c_{2}+b_{3} p_{1}\right)-\frac{1}{4}\left(c_{3} b_{2}-q_{1} b_{2}-c_{3} a_{3}+q_{1} a_{3}\right)-\frac{3}{4}\left(c_{3} d_{1}-q_{1} d_{1}\right)+d_{2} q_{2}- \\
& d_{2} p_{3}+p_{2} d_{3}-d_{3} q_{3} . \\
R_{2424}= & -\frac{1}{2}\left(c_{2} p_{1}+q_{2} p_{3}\right)+\frac{1}{4}\left(c_{2}^{2}-q_{2}^{2}\right)-\frac{3}{4}\left(p_{1}^{2}-p_{3}^{2}\right)-p_{2}^{2}, \\
R_{2434}= & \frac{1}{4}\left(c_{2} q_{1}+c_{2} c_{3}-p_{1} c_{3}\right)+\frac{3}{4} p_{1} q_{1}+p_{2} q_{2}-p_{3} q_{3} . \\
R_{3434}= & -\frac{1}{2}\left(c_{3} q_{1}+q_{2} p_{3}\right)-\frac{1}{4}\left(c_{3}^{2}+p_{3}^{2}\right)+\frac{3}{4}\left(q_{1}^{2}+q_{2}^{2}\right)-q_{3}^{2} .
\end{aligned}
$$

A long but straightforward calculation then gives by contraction the components for the Ricci tensor with respect to $\left\{e_{1}, e_{2}, e_{3}, e_{4}\right\}$ :

$$
\begin{aligned}
& \rho\left(e_{1}, e_{1}\right)=\frac{1}{2}\left(c_{3}{ }^{2}-q_{1}{ }^{2}+b_{2}{ }^{2}-d_{1}{ }^{2}+a_{3}{ }^{2}-c_{2}{ }^{2}+p_{1}{ }^{2}\right)-b_{3}{ }^{2}-a_{1}{ }^{2} \\
& -c_{1}^{2}-a_{2}^{2}+b_{1}^{2}-b_{2} a_{3}-c_{1} q_{3}+a_{1} d_{3}+b_{1} d_{2}-c_{1} p_{2} \text {, } \\
& \rho\left(e_{1}, e_{2}\right)=\frac{1}{2}\left(p_{3} c_{3}-q_{2} q_{1}-q_{3} c_{2}-q_{3} p_{1}\right)-d_{2} a_{3}+b_{2} d_{2}+a_{2} d_{3} \\
& +b_{1} d_{1}-b_{3} d_{3}-c_{1} p_{1}-p_{2} c_{2} \text {, } \\
& \rho\left(e_{1}, e_{3}\right)=\frac{1}{2}\left(p_{2} c_{3}-p_{2} q_{1}-c_{2} q_{2}-p_{1} p_{3}\right)-d_{2} b_{3}+d_{3} b_{2}-a_{3} d_{3} \\
& +a_{1} d_{1}+a_{2} d_{2}+c_{3} q_{3}-c_{1} q_{1} \text {, } \\
& \rho\left(e_{1}, e_{4}\right)=\frac{1}{2}\left(b_{2} p_{3}+a_{3} q_{2}-d_{2} q_{1}-d_{2} c_{3}-b_{2} q_{2}-a_{3} p_{3}+d_{3} c_{2}\right. \\
& \left.-d_{3} p_{1}\right)+a_{1} p_{1}+a_{2} p_{2}-b_{1} q_{1}+b_{3} q_{3} \text {, } \\
& \rho\left(e_{2}, e_{2}\right)=\frac{1}{2}\left(p_{3}{ }^{2}-q_{2}{ }^{2}-b_{2}{ }^{2}+d_{1}{ }^{2}+a_{3}{ }^{2}+c_{2}{ }^{2}-p_{1}{ }^{2}\right)-d_{3}{ }^{2}-p_{2}{ }^{2} \\
& -a_{2}^{2}+d_{2}^{2}-a_{1}^{2}-a_{2} b_{3}-p_{2} q_{3}+d_{1} a_{3}+b_{1} d_{2}-c_{1} p_{2} \text {, } \\
& \rho\left(e_{2}, e_{3}\right)=\frac{1}{2}\left(c_{1} p_{3}-c_{1} q_{2}-c_{2} c_{3}-p_{1} q_{1}\right)-b_{1} d_{3}+b_{3} d_{1}+a_{3} b_{3} \\
& -a_{1} b_{1}-a_{2} b_{2}+p_{3} q_{3}-p_{2} q_{2} \text {, } \\
& \rho\left(e_{2}, e_{4}\right)=\frac{1}{2}\left(c_{3} d_{1}-q_{1} a_{3}+c_{3} a_{3}-b_{1} q_{2}-b_{1} p_{3}-q_{1} d_{1}-b_{3} c_{2}\right. \\
& \left.+b_{3} p_{1}\right)-a_{1} c_{1}-a_{2} c_{2}-d_{2} q_{2}+d_{3} q_{3} \text {, } \\
& \rho\left(e_{3}, e_{3}\right)=\frac{1}{2}\left(p_{3}^{2}-q_{2}^{2}+c_{3}^{2}-q_{1}^{2}-b_{2}^{2}-d_{1}^{2}+a_{3}^{2}\right)+q_{3}^{2}+d_{3}^{2} \\
& -d_{2}{ }^{2}+b_{3}{ }^{2}-b_{1}{ }^{2}+a_{2} b_{3}+p_{2} q_{3}-b_{2} d_{1}+c_{1} q_{3}-a_{1} d_{3} \text {, } \\
& \rho\left(e_{3}, e_{4}\right)=\frac{1}{2}\left(a_{2} q_{1}+a_{2} c_{3}-a_{1} q_{2}-a_{1} p_{3}-b_{2} p_{1}-d_{1} p_{1}-b_{2} c_{2}\right. \\
& \left.-d_{1} c_{2}\right)-b_{1} c_{1}+b_{3} c_{3}-d_{2} p_{2}+d_{3} p_{3} \text {, } \\
& \rho\left(e_{4}, e_{4}\right)=\frac{1}{2}\left(q_{1}{ }^{2}+c_{3}{ }^{2}-p_{1}{ }^{2}-c_{2}{ }^{2}+q_{2}{ }^{2}+p_{3}{ }^{2}\right)-c_{1}{ }^{2}-q_{3}{ }^{2}-p_{2}{ }^{2} \\
& -c_{2} p_{1}-c_{3} q_{1}-p_{3} q_{2} \text {. }
\end{aligned}
$$

The Lie group $(G, g)$ satisfies the Ricci cyclic-parallel property if and only if the Equation (1.2) holds. By solving the algebraic systems (3.2) and (1.1) and eliminating the Ricci parallel solutions, we conclude that the generally non-isomorphic solutions are exactly the sets 1)-14) of Lie algebras listed in case a) of the statement of the theorem. In the cases 1)-6) we have that $\left[\mathfrak{g}_{3}, \mathfrak{g}_{3}\right]$ is onedimensional and comparing with the classification of three-dimensional Lorentzian Lie algebras given in [18], we see that $\mathfrak{g}_{3}$ is the Lie algebra of the Heisenberg group. About the cases 7)-8) and 9)-14) we have, $\left[\mathfrak{g}_{3}, \mathfrak{g}_{3}\right]$ is two-dimensional and trivial respectively. Thus, the Lie algebra $\mathfrak{g}_{3}$ corresponds to the Lie group $E(2)$ or $E(1,1)$ and $\mathbb{R}^{3}$ respectively.

Case (b). Similar to the previous case, we treat this case with the obvious difference that $e_{4}$ is now a time-like vector acting on a three-dimensional Riemannian Lie algebra. 
With respect to the pseudo-orthonormal basis $\left\{e_{1}, e_{2}, e_{3}, e_{4}\right\}$ with $e_{4}$ time-like the components of the Levi-Civita connection are now given by

$$
\begin{gathered}
\Lambda_{1}=\left(\begin{array}{cccc}
0 & a_{1} & b_{1} & c_{1} \\
-a_{1} & 0 & \frac{b_{2}+d_{1}-a_{3}}{2} & \frac{c_{2}+p_{1}}{2} \\
-b_{1} & \frac{a_{3}-d_{1}-b_{2}}{2} & 0 & \frac{c_{3}+q_{1}}{2} \\
c_{1} & \frac{c_{2}+p_{1}}{2} & \frac{c_{3}+q_{1}}{2} & 0
\end{array}\right), \Lambda_{2}=\left(\begin{array}{cccc}
0 & a_{2} & \frac{d_{1}+b_{2}+a_{3}}{2} & \frac{c_{2}+p_{1}}{2} \\
-a_{2} & 0 & d_{2} & p_{2} \\
-\frac{a_{3}+b_{2}+d_{1}}{2} & -d_{2} & 0 & \frac{p_{3}+q_{2}}{2} \\
\frac{c_{2}+p_{1}}{2} & p_{2} & \frac{p_{3}+q_{2}}{2} & 0
\end{array}\right), \\
\Lambda_{3}=\left(\begin{array}{ccccc}
0 & \frac{-d_{1}+a_{3}+b_{2}}{2} & b_{3} & \frac{c_{3}+q_{1}}{2} \\
\frac{-b_{2}-a_{3}+d_{1}}{2} & 0 & d_{3} \frac{p_{3}+q_{2}}{2} \\
-b_{3} & -d_{3} & 0 & q_{3} \\
\frac{c_{3}+q_{1}}{2} & \frac{p_{3}+q_{2}}{2} & q_{3} & 0
\end{array}\right), \Lambda_{4}=\left(\begin{array}{cccc}
0 & \frac{-p_{1}+c_{2}}{2} & \frac{-q_{1}+c_{3}}{2} & 0 \\
\frac{-c_{2}+p_{1}}{2} & 0 & \frac{-q_{2}+p_{3}}{2} & 0 \\
\frac{-c_{3}+q_{1}}{2} & \frac{-p_{3}+q_{2}}{2} & 0 & 0 \\
0 & 0 & 0 & 0
\end{array}\right)
\end{gathered}
$$

and the components of the Ricci tensor, deduced as in the previous case, are the following:

$$
\begin{aligned}
& \rho\left(e_{1}, e_{1}\right)=\frac{1}{2}\left(d_{1}^{2}-b_{2}^{2}-a_{3}^{2}+c_{2}^{2}-p_{1}^{2}+c_{3}^{2}-q_{1}^{2}\right)+c_{1}^{2}-a_{1}^{2}-b_{1}^{2}-a_{2}^{2}-b_{3}^{2} \\
& +c_{1} p_{2}-b_{1} d_{2}-b_{2} a_{3}+a_{1} d_{3}+c_{1} q_{3} \text {, } \\
& \rho\left(e_{1}, e_{2}\right)=\frac{1}{2}\left(q_{3} c_{2}+q_{3} p_{1}+c_{3} p_{3}-q_{1} q_{2}\right) \\
& +a_{2} d_{3}-d_{2} a_{3}-d_{2} b_{2}-b_{1} d_{1}-b_{3} d_{3}+c_{1} p_{1}+c_{2} p_{2} \text {, } \\
& \rho\left(e_{1}, e_{3}\right)=\frac{1}{2}\left(p_{2} c_{3}+p_{2} q_{1}+c_{2} q_{2}-p_{1} p_{3}\right) \\
& -d_{2} b_{3}+a_{3} d_{3}+d_{3} b_{2}+a_{1} d_{1}+a_{2} d_{2}+c_{1} q_{1}+c_{3} q_{3} \text {, } \\
& \rho\left(e_{1}, e_{4}\right)=\frac{1}{2}\left(-d_{2} c_{3}+d_{2} q_{1}+b_{2} p_{3}+b_{2} q_{2}+a_{3} p_{3}+a_{3} q_{2}+d_{3} c_{2}-d_{3} p_{1}\right) \\
& +a_{1} p_{1}+a_{2} p_{2}+b_{1} q_{1}+b_{3} q_{3} \text {, } \\
& \rho\left(e_{2}, e_{2}\right)=\frac{1}{2}\left(-d_{1}^{2}+b_{2}^{2}-a_{3}^{2}-c_{2}^{2}+p_{1}^{2}+p_{3}^{2}-q_{2}^{2}\right)-a_{1}^{2}-a_{2}^{2}+p_{2}^{2}-d_{2}^{2}-d_{3}^{2} \\
& +c_{1} p_{2}-b_{1} d_{2}+d_{1} a_{3}+p_{2} q_{3}-a_{2} b_{3} \text {, } \\
& \rho\left(e_{2}, e_{3}\right)=\frac{1}{2}\left(c_{1} p_{3}+c_{1} q_{2}-c_{2} c_{3}+p_{1} q_{1}\right) \\
& -b_{1} d_{3}-a_{3} b_{3}+b_{3} d_{1}-a_{1} b_{1}-a_{2} b_{2}+p_{2} q_{2}+p_{3} q_{3} \text {, } \\
& \rho\left(e_{2}, e_{4}\right)=\frac{1}{2}\left(-b_{1} p_{3}+b_{1} q_{2}-a_{3} q_{1}+d_{1} q_{1}-a_{3} c_{3}+d_{1} c_{3}+b_{3} p_{1}-b_{3} c_{2}\right) \\
& -a_{1} c_{1}-a_{2} c_{2}+d_{2} q_{2}+d_{3} q_{3} \text {, } \\
& \rho\left(e_{3}, e_{3}\right)=\frac{1}{2}\left(-d_{1}^{2}-b_{2}^{2}+a_{3}^{2}-c_{3}^{2}+q_{1}^{2}-p_{3}^{2}+q_{2}^{2}\right)-b_{1}^{2}-b_{3}^{2}-d_{2}^{2}-d_{3}^{2}+q_{3}^{2} \\
& -d_{1} b_{2}+a_{1} d_{3}+c_{1} q_{3}+p_{2} q_{3}-a_{2} b_{3} \text {, } \\
& \rho\left(e_{3}, e_{4}\right)=\frac{1}{2}\left(-a_{1} q_{2}+a_{1} p_{3}-b_{2} p_{1}-d_{1} p_{1}-b_{2} c_{2}-d_{1} c_{2}+a_{2} q_{1}-a_{2} c_{3}\right) \\
& -b_{1} c_{1}-b_{3} c_{3}-d_{2} p_{2}-d_{3} p_{3} \text {, } \\
& \rho\left(e_{4}, e_{4}\right)=\frac{1}{2}\left(-p_{1}^{2}-c_{2}^{2}-q_{1}^{2}-c_{3}^{2}-q_{2}^{2}-p_{3}^{2}\right) \\
& -c_{1}^{2}-p_{2}^{2}-q_{3}^{2}-c_{2} p_{1}-c_{3} q_{1}-p_{3} q_{2} \text {. }
\end{aligned}
$$

We then determined the set of all solutions of (3.2) and (1.1). The non-Ricci parallel and generally non-isomorphic solutions are exactly those listed in the items 15)-19), specially, for the cases 15)16 we have $\left[\mathfrak{g}_{3}, \mathfrak{g}_{3}\right]$ is three-dimensional, for 17)-18) $\left[\mathfrak{g}_{3}, \mathfrak{g}_{3}\right]$ is two-dimensional and in the case 19) $\left[\mathfrak{g}_{3}, \mathfrak{g}_{3}\right]=0$, which shows that the corresponding Lie group is Heisenberg, $E(2)$ or $E(1,1)$ and $\mathbb{R}^{3}$ respectively. To note that in some of the solutions, $[\mathfrak{g}, \mathfrak{g}]$ is two-dimensional and the Lie algebra $\mathfrak{g}$, which corresponds to a semi-direct product $\mathfrak{r} \ltimes \mathfrak{g}_{3}$, with $\mathfrak{g}_{3}$ Riemannian, also corresponds to a semi-direct product $\mathfrak{r}^{\prime} \ltimes \mathfrak{g}_{3}^{\prime}$, where $\mathfrak{g}_{3}^{\prime}$ is Lorentzian. Hence, such example is already included in the case a).

For example, an explicit solution for this case is given by

$$
\left[e_{1}, e_{2}\right]=A e_{2}, \quad\left[e_{1}, e_{3}\right]=\frac{A}{2} e_{3}, \quad\left[e_{1}, e_{4}\right]=A e_{2},
$$


for some real constant $A$. Thus, $[\mathfrak{g}, \mathfrak{g}]=\operatorname{Span}\left(e_{2}, e_{3}\right)$, and the time-like vector $e_{4}$ acts as a derivation on the Riemannian Lie algebra $\mathfrak{g}_{3}=\operatorname{Span}\left(e_{1}, e_{2}, e_{3}\right)$. On the other hand, the above equations yield that the space-like vector $e_{1}$ also acts as a derivation on the Lorentzian Lie algebra $\mathfrak{g}_{3}^{\prime}=\operatorname{Span}\left(e_{2}, e_{3}, e_{4}\right)$, which is the Lie algebra of $\mathbb{R}^{3}$. Therefore, this example is already included in the case a).

Case (c). In this case, with respect to the basis $\left\{e_{1}, e_{2}, e_{3}, e_{4}\right\}$, where the non-zero components of the metric are $g\left(e_{1}, e_{1}\right)=g\left(e_{2}, e_{2}\right)=g\left(e_{3}, e_{4}\right)=g\left(e_{4}, e_{3}\right)=1$, the Levi-Civita connection is described by

$$
\begin{gathered}
\Lambda_{1}=\left(\begin{array}{cccc}
0 & a_{1} & b_{1} & c_{1} \\
-a_{1} & 0 & \frac{b_{2}+d_{1}}{2} & \frac{c_{2}+p_{1}-a_{3}}{2} \\
-c_{1} \frac{a_{3}-p_{1}-c_{2}}{2} & \frac{b_{3}-q_{1}}{2} & 0 \\
-b_{1} & -\frac{d_{1}+b_{2}}{2} & 0 & \frac{q_{1}-b_{3}}{2}
\end{array}\right), \Lambda_{2}=\left(\begin{array}{cccc}
0 & a_{2} & \frac{b_{2}+d_{1}}{2} & \frac{p_{1}+c_{2}+a_{3}}{2} \\
-a_{2} & 0 & d_{2} & p_{2} \\
-\frac{a_{3}+c_{2}+p_{1}}{2}-p_{2} & \frac{d_{3}-q_{2}}{2} & 0 \\
-\frac{d_{1}+b_{2}}{2} & -d_{2} & 0 & \frac{q_{2}-d_{3}}{2}
\end{array}\right), \\
\Lambda_{3}=\left(\begin{array}{cccc}
0 & \frac{-d_{1}+b_{2}}{2} & 0 & \frac{q_{1}+b_{3}}{2} \\
\frac{-b_{2}+d_{1}}{2} & 0 & 0 & \frac{q_{2}+d_{3}}{2} \\
-\frac{b_{3}+q_{1}}{2} & -\frac{d_{3}+q_{2}}{2} & 0 & 0 \\
0 & 0 & 0 & 0
\end{array}\right), \Lambda_{4}=\left(\begin{array}{cccc}
0 & \frac{-p_{1}+a_{3}+c_{2}}{2} \frac{b_{3}-q_{1}}{2} c_{3} \\
\frac{-c_{2}-a_{3}+p_{1}}{2} & 0 & \frac{d_{3}-q_{2}}{2} & p_{3} \\
-c_{3} & -p_{3} & -q_{3} & 0 \\
\frac{q_{1}-b_{3}}{2} & \frac{q_{2}-d_{3}}{2} & 0 & q_{3}
\end{array}\right)
\end{gathered}
$$

and the Ricci tensor is then determined by the following components:

$$
\begin{aligned}
\rho\left(e_{1}, e_{1}\right)= & -\frac{1}{2}\left(q_{1}^{2}+b_{3}^{2}\right)-a_{2}^{2}-a_{1}^{2}-2 b_{1} c_{1} \\
& -b_{1} p_{2}-c_{1} d_{2}-b_{2} a_{3}-b_{2} c_{2}+d_{1} p_{1}+a_{1} d_{3}-b_{1} q_{3}, \\
\rho\left(e_{1}, e_{2}\right)= & -\frac{1}{2}\left(q_{2} q_{1}+d_{3} b_{3}+q_{3} b_{2}+q_{3} d_{1}\right)-b_{1} p_{1}-p_{2} b_{2}-c_{1} d_{1}-d_{2} c_{2}-d_{2} a_{3}+a_{2} d_{3}, \\
\rho\left(e_{1}, e_{3}\right)= & -\frac{1}{2}\left(d_{2} b_{3}+d_{2} q_{1}+d_{1} d_{3}+b_{2} q_{2}\right)+b_{2} d_{3}+a_{1} d_{1}+a_{2} d_{2}-b_{1} q_{1}, \\
\rho\left(e_{1}, e_{4}\right)= & \frac{1}{2}\left(p_{2} q_{1}-p_{2} b_{3}+c_{2} q_{2}+c_{2} d_{3}+a_{3} q_{2}+a_{3} d_{3}+p_{3} b_{2}+p_{3} d_{1}\right) \\
& -d_{2} c_{3}+a_{1} p_{1}+a_{2} p_{2}+c_{1} q_{1}, \\
\rho\left(e_{2}, e_{2}\right)= & -\frac{1}{2}\left(q_{2}^{2}+d_{3}^{2}\right)-a_{2}^{2}-a_{1}^{2}-b_{1} p_{2}-c_{1} d_{2}+b_{2} c_{2} \\
& +d_{1} a_{3}-d_{1} p_{1}-2 d_{2} p_{2}-a_{2} b_{3}-d_{2} q_{3}, \\
\rho\left(e_{2}, e_{3}\right)= & -\frac{1}{2}\left(b_{1} d_{3}+b_{1} q_{2}+d_{1} q_{1}+b_{2} b_{3}\right)+d_{1} b_{3}-a_{1} b_{1}-a_{2} b_{2}-d_{2} q_{2}, \\
\rho\left(e_{2}, e_{4}\right)= & \frac{1}{2}\left(c_{1} q_{2}-c_{1} d_{3}-a_{3} b_{3}+p_{1} b_{3}-a_{3} q_{1}+p_{1} q_{1}+c_{3} d_{1}+c_{3} b_{2}\right) \\
& -b_{1} p_{3}-a_{1} c_{1}-a_{2} c_{2}+p_{2} q_{2}, \\
\rho\left(e_{3}, e_{3}\right)= & -\frac{1}{2}\left(d_{1}^{2}+b_{2}^{2}\right)-b_{1}^{2}-d_{2}^{2}-d_{1} b_{2}, \\
\rho\left(e_{3}, e_{4}\right)= & -\frac{1}{2}\left(b_{2} p_{1}+b_{2} c_{2}+d_{1} p_{1}+d_{1} c_{2}+a_{1} q_{2}-a_{1} d_{3}-q_{1}^{2}+b_{3}^{2}-a_{2} q_{1}+a_{2} b_{3}-q_{2}^{2}+d_{3}^{2}\right) \\
& -b_{1} c_{1}-b_{1} q_{3}-d_{2} p_{2}-d_{2} q_{3}, \\
\rho\left(e_{4}, e_{4}\right)= & \frac{1}{2}\left(-p_{1}^{2}+a_{3}^{2}-c_{2}^{2}\right)-c_{1}^{2}-p_{2}^{2}+a_{1} p_{3}+c_{1} q_{3} \\
& -c_{3} q_{1}-p_{1} c_{2}-a_{2} c_{3}+p_{2} q_{3}-p_{3} q_{2} .
\end{aligned}
$$

Also in this case, we used the above equations and solved simultaneously (3.2) and (1.1). As in the proof of the case b), for some solutions there also exists a space-like vector acting as a derivation over a three-dimensional Lorentzian Lie algebra. Thus, these solutions were already listed in case a). The remaining solutions are the ones we listed in the cases 20)-27). In the cases 20)-21), we have that $\left[\mathfrak{g}_{3}, \mathfrak{g}_{3}\right]$ is one-dimensional, and the three-dimensional Lie algebra is the one of the Heisenberg group. On the other hand, in the case $\mathbf{2 2}$ ) we have that $\left[\mathfrak{g}_{3}, \mathfrak{g}_{3}\right]$ is two-dimensional, in the cases $\mathbf{2 3}$ )26) we have that $\left[\mathfrak{g}_{3}, \mathfrak{g}_{3}\right]$ is three-dimensional and in the case 27) we have that $\left[\mathfrak{g}_{3}, \mathfrak{g}_{3}\right]=0$. Thus, they correspond to semi-direct products of type $\mathbb{R} \ltimes \mathbb{E}(2)$ or $\mathbb{R} \ltimes \mathbb{E}(1,1), \mathbb{R} \ltimes \widetilde{S L}(2, \mathbb{R})$ or $\mathbb{R} \ltimes S U(2)$ and $\mathbb{R} \ltimes \mathbb{R}^{3}$ respectively. 
A pseudo-Riemannian manifold $(M, g)$ is said to be a Walker manifold if it admits a null (that is, degenerate) parallel distribution [19,20]. Walker structures do not have any Riemannian counterpart, and are responsible for several of the most interesting differences between Riemannian and pseudo-Riemannian geometries. A wide survey on Walker geometry may be found in [6]. We now investigate the Walker examples through the Einstein-like Lorentzian Lie groups of class $\mathscr{A}$ which are not Ricci parallel.

Theorem 3.2. Among the non-Ricci parallel four-dimensional Einstein-like Lorentzian Lie groups of class $\mathscr{A}$, as classified in Theorem 3.1 up to isometries, the Walker examples are

(a) case 1) with $A=\frac{\varepsilon B C}{\sqrt{C^{2}-D^{2}-B^{2}}}$. In this case $X=D e_{1}-\varepsilon \sqrt{C^{2}-D^{2}-B^{2}} e_{2}-C e_{3}+B e_{4}$ is a left-invariant null parallel line field.

(b) case 5) with $B=\varepsilon C, D=\varepsilon E$. In this case $X=e_{3}-\varepsilon e_{4}$ is a left-invariant null parallel line field.

(c) case 10) with $A=\varepsilon C$. In this case $X=e_{1}-\varepsilon e_{3}$ is a left-invariant null parallel line field.

(d) case 14) with $B=0$. In this case $X=e_{1}+e_{3}$ is a left-invariant null parallel line field.

(e) case 20) with $F=\frac{-2 A B C(E+D)+C^{2} E^{2}+A^{2}(E+D)^{2}}{2 A C^{2}}$. In this case $X=-\frac{E+D}{C} e_{1}+\frac{E}{A} e_{2}-$ $\frac{C^{2} E^{2}+A^{2}(D+E)^{2}}{2 A^{2} C^{2}} e_{3}+e_{4}$ is a left-invariant null parallel line field.

(f) case 25), in this case $X=e_{3}$ is always a left-invariant null parallel line field.

(g) case 26), in this case $X=e_{3}$ is always a left-invariant null parallel line field.

Proof. The proof is based on the case-by-case considering the examples of class $\mathscr{A}$, which are listed in the Theorem 3.1. Let $G$ is isometric to $\mathbb{R} \ltimes H$, where $H$ is the Heisenberg group, and $\mathfrak{g}$ is described by the brackets of the case 1). Using the Koszul formula the Levi-Civita connection with respect to the pseudo-orthonormal basis $\left\{e_{i}\right\}_{i=1}^{4}$, with $e_{3}$ time-like, is

$$
\begin{aligned}
& \Lambda_{1}=\left(\begin{array}{cccc}
0 & 0 & 0 & 0 \\
0 & 0 & \frac{A}{2} & \frac{A C}{2 B} \\
0 & \frac{A}{2} & 0 & \frac{C}{2} \\
0 & -\frac{A C}{2 B} & \frac{C}{2} & 0
\end{array}\right), \quad \Lambda_{2}=\left(\begin{array}{cccc}
0 & 0 & \frac{A}{2} & \frac{A C}{2 B} \\
0 & 0 & 0 & 0 \\
\frac{A}{2} & 0 & 0 & -\frac{A D}{2 B} \\
-\frac{A C}{2 B} & 0-\frac{A D}{2 B} & 0
\end{array}\right), \\
& \Lambda_{3}=\left(\begin{array}{cccc}
0 & \frac{A}{2} & -B & -\frac{C}{2} \\
-\frac{A}{2} & 0 & 0 & \frac{A D}{2 B} \\
-B & 0 & 0 & D \\
\frac{C}{2} & -\frac{A D}{2 B} & D & 0
\end{array}\right), \Lambda_{4}=\left(\begin{array}{cccc}
0 & \frac{A C}{2 B} & -\frac{C}{2} & 0 \\
-\frac{A C}{2 B} & 0 & -\frac{A D}{2 B} & 0 \\
-\frac{C}{2} & -\frac{A D}{2 B} & 0 & 0 \\
0 & 0 & 0 & 0
\end{array}\right) .
\end{aligned}
$$

Now, suppose that $X=x_{1} e_{1}+x_{2} e_{2}+x_{3} e_{3}+x_{4} e_{4}$ generates a null parallel line field on the Lie group $G$ of the case 1) of Theorem 3.1. Since $X$ is null, so $x_{1}^{2}+x_{2}^{2}-x_{3}^{2}+x_{4}^{2}=0$ and $X$ is parallel if and only if there exist a one form $\omega=\omega_{1} \theta_{1}+\omega_{2} \theta_{2}+\omega_{3} \theta_{3}+\omega_{4} \theta_{4}$ such that $\nabla_{e_{i}} X=\omega\left(e_{i}\right) X, i=1, \ldots, 4$, where $\left\{\theta_{i}\right\}_{i=1}^{4}$ is the dual basis of $\left\{e_{i}\right\}_{i=1}^{4}$. Using description of the Levi-Civita connection the only solution of this system of equations is:

$$
A=\frac{\varepsilon B C}{\sqrt{C^{2}-B^{2}-D^{2}}}, x_{1}=\frac{D}{B} x_{4}, x_{2}=-\frac{\varepsilon \sqrt{C^{2}-B^{2}-D^{2}}}{B} x_{4}, x_{3}=-\frac{C}{B} x_{4}, \omega=0,
$$

which specifies the case (a) of the statement of theorem. 


\section{Examples of class $\mathscr{B}$}

After excluding the Ricci parallel cases, the list of Codazzi Ricci tensor Lorentzian Lie groups is given in the following theorem:

Theorem 4.1. Let $G$ be a four-dimensional simply connected Lie group equipped with a left invariant Lorentzian metric $g$. If $(G, g)$ belongs to class $\mathscr{B}$ (and not Ricci parallel), then the Lie algebra $\mathfrak{g}$ of $G$ is isometric to $\mathfrak{g}=\mathfrak{r} \ltimes \mathfrak{g}_{3}$, where $\mathfrak{g}_{3}=\operatorname{span}\left\{e_{1}, e_{2}, e_{3}\right\}$ and $\mathfrak{r}=\operatorname{span}\left\{e_{4}\right\}$, and one of the following cases occurs.

(a) $\left\{e_{i}\right\}_{i=1}^{4}$ is a pseudo-orthonormal basis with $e_{3}$ time-like. In this case, $G$ is isometric to one of the following semi-direct products $\mathbb{R} \ltimes G_{3}$.

a1) $\mathbb{R} \ltimes E(2)$ or $\mathbb{R} \ltimes E(1,1)$, with $\mathfrak{g}$ is described by one of the following sets of conditions:

1) $\left[e_{1}, e_{2}\right]=-3 A e_{1}+\varepsilon 2 A e_{3},\left[e_{2}, e_{3}\right]=A e_{3}$,

a2) $\mathbb{R} \ltimes \mathbb{R}^{3}$, with $\mathfrak{g}$ is described by one of the following sets of conditions:

2) $\left[e_{1}, e_{4}\right]=(\varepsilon 4 A+3 B) e_{1}-(3 A+\varepsilon 2 B) e_{3},\left[e_{3}, e_{4}\right]=A e_{1}+B e_{3}, B+\varepsilon A \neq 0, B+\varepsilon 2 A \neq 0$,

3) $\left[e_{1}, e_{4}\right]=-B e_{1}+(A+\delta 2 B) e_{3},\left[e_{2}, e_{4}\right]=(\varepsilon \sqrt{5}+\delta)(A+\delta B) e_{2},\left[e_{3}, e_{4}\right]=A e_{1}+B e_{3}, B \neq$ $0, B+\delta A \neq 0$,

4) $\left[e_{1}, e_{4}\right]=(\varepsilon 4 A+3 B) e_{1}-(3 A+\varepsilon 2 B) e_{3},\left[e_{2}, e_{4}\right]=2(\varepsilon A+B) e_{2},\left[e_{3}, e_{4}\right]=A e_{1}+B e_{3}, B+$ $\varepsilon A \neq 0, B+\varepsilon 2 A \neq 0$,

5) $\left[e_{1}, e_{4}\right]=\varepsilon 4 \sqrt{A^{2}-B^{2}} e_{1}+3 B e_{2}-3 A e_{3},\left[e_{2}, e_{4}\right]=B e_{1},\left[e_{3}, e_{4}\right]=A e_{1}, A \pm B \neq 0$,

6) $\left[e_{1}, e_{4}\right]=-A e_{1}-2 A e_{3},\left[e_{2}, e_{4}\right]=(\varepsilon \sqrt{5}+1) A e_{2},\left[e_{3}, e_{4}\right]=A e_{3}$,

7) $\left[e_{2}, e_{4}\right]=\frac{3 \varepsilon}{2} A e_{2}+A e_{3},\left[e_{3}, e_{4}\right]=\frac{\varepsilon}{2} A e_{3}$,

8) $\left[e_{1}, e_{4}\right]=(\varepsilon \sqrt{5}+1) A e_{1},\left[e_{2}, e_{4}\right]=-A e_{2}-\delta 2 A e_{3},\left[e_{3}, e_{4}\right]=A e_{3}$,

9) $\left[e_{1}, e_{4}\right]=2 A e_{1},\left[e_{2}, e_{4}\right]=3 A e_{2}+\delta 2 A e_{3},\left[e_{3}, e_{4}\right]=A e_{3}$,

(c) $\left\{e_{i}\right\}_{i=1}^{4}$ is a basis with the inner product completely determined by $g\left(e_{1}, e_{1}\right)=g\left(e_{2}, e_{2}\right)=$ $g\left(e_{3}, e_{4}\right)=g\left(e_{4}, e_{3}\right)=1$ and $g\left(e_{i}, e_{j}\right)=0$ otherwise. In this case, $G$ is isometric to one of the following semi-direct products $\mathbb{R} \ltimes G_{3}$.

c1) $\mathbb{R} \ltimes H$, where $H$ is the Heisenberg group and $\mathfrak{g}$ is described by one of the following sets of conditions:

10) $\left[e_{1}, e_{2}\right]=A e_{3},\left[e_{1}, e_{4}\right]=B e_{1}+C e_{2}+D e_{3},\left[e_{2}, e_{4}\right]=E e_{1}+F e_{2}+G e_{3},\left[e_{3}, e_{4}\right]=(F+$ $B) e_{3}, B+F \neq 0, F^{2}+(A \pm(C+E))^{2} \neq 0,4 B F+A^{2}-(E+C)^{2} \neq 0$,

c2) $\mathbb{R} \ltimes E(2)$ or $\mathbb{R} \ltimes E(1,1)$, with $\mathfrak{g}$ is described by one of the following sets of conditions:

11) $\left[e_{1}, e_{2}\right]=-\frac{1}{2} A e_{1}+\frac{1}{2} B e_{2}, \quad\left[e_{1}, e_{3}\right]=B e_{3}, \quad\left[e_{1}, e_{4}\right]=$ $\frac{2 C B+A D}{2 A} e_{1}-\frac{B(2 C B+A D)}{2 A^{2}} e_{2}+\frac{B\left(4 C^{2} B^{2}+4 A D C B+A^{2} D^{2}+4 A^{2} C^{2}\right)}{2 A^{4}} e_{3},\left[e_{2}, e_{3}\right]=A e_{3},\left[e_{2}, e_{4}\right]=C e_{1}-$ $\frac{C B}{A} e_{2}+\frac{4 C^{2} B^{2}+4 A D C B+A^{2} D^{2}+4 A^{2} C^{2}}{2 A^{3}} e_{3},\left[e_{3}, e_{4}\right]=D e_{3}, B^{2}+C^{2} \neq 0, A B D+2 C\left(A^{2}+B^{2}\right) \neq 0$,

12) $\left[e_{1}, e_{2}\right]=$ $-\frac{1}{2} A e_{1}+\frac{1}{2} B e_{2}+C e_{3},\left[e_{1}, e_{3}\right]=B e_{3},\left[e_{1}, e_{4}\right]=D e_{1}-\frac{D B}{A} e_{2}+\frac{2\left(B E^{2}+D^{2} B-D C A\right)}{A^{2}} e_{3},\left[e_{2}, e_{3}\right]=$ $A e_{3},\left[e_{2}, e_{4}\right]=E e_{1}-\frac{E B}{A} e_{2}+\frac{2\left(E^{2}-E C+D^{2}\right)}{A} e_{3},\left[e_{3}, e_{4}\right]=\frac{2(A D-B E)}{A} e_{3}, A C-A E-D B \neq 0$, 
c3) $\mathbb{R} \ltimes \widetilde{S L}(2, \mathbb{R})$ or $\mathbb{R} \ltimes S U(2)$ with $\mathfrak{g}$ is described by one of the following sets of conditions:

13) $\left[e_{1}, e_{2}\right]=A e_{2}+B e_{3}, \quad\left[e_{1}, e_{3}\right]=-C e_{1}-A e_{3}, \quad\left[e_{1}, e_{4}\right]=-\frac{A^{2}}{2 C} e_{1}-\frac{A^{3}}{2 C^{2}} e_{3}, \quad\left[e_{2}, e_{3}\right]=$ $C e_{2},\left[e_{2}, e_{4}\right]=-\frac{A^{2}}{2 C} e_{2}-\frac{B A}{C} e_{3},\left[e_{3}, e_{4}\right]=A e_{1}+\frac{A^{2}}{C} e_{3}, B \neq 0$,

c4) $\mathbb{R} \ltimes \mathbb{R}^{3}$, with $\mathfrak{g}$ is described by one of the following sets of conditions:

14) $\left[e_{1}, e_{4}\right]=A e_{1}+B e_{2}+C e_{3},\left[e_{2}, e_{4}\right]=D e_{1}+E e_{2}+F e_{3},\left[e_{3}, e_{4}\right]=G e_{3}, G \neq 0, G-$ $\frac{(B+D)^{2}+2 A^{2}+2 E^{2}}{2(A+E)} \neq 0$,

Proof. The proof is similar to the Theorem 3.1, but in this case, we require that the Ricci tensor is Codazzi, i.e. the Equation (1.3) must be satisfied, noting that we must discard the Ricci parallel examples.

Let $(G, g)$ be a four-dimensional Lorentzian Lie group. The general form of the semi-direct product Lie algebra $\mathfrak{g}=\mathfrak{r} \ltimes \mathfrak{g}_{3}$ showed in the Equation (3.1), where $\mathfrak{g}_{3}$ is spanned by $\left\{e_{1}, e_{2}, e_{3}\right\}$, $\mathfrak{r}=\operatorname{Span}\left(e_{4}\right), \mathfrak{r}$ acts on $\mathfrak{g}_{3}$ and the Lorentzian inner product is described by one of the cases (a), (b) or (c) of the Proposition 2.1. The Equations (3.2) are required for establishing the Jacobi identity. To specify the examples of the class $\mathscr{B}$, we treat different possibilities of the inner product i.e. cases (a), (b) and (c).

Let the inner product be of type (a), by using the Equations (3.4), excluding the Ricci parallel solutions, the generally non-isomorphic examples of class $\mathscr{B}$ are exactly the cases 1)-9) which are listed in the statement.

With respect to the basis $\left\{e_{1}, e_{2}, e_{3}, e_{4}\right\}$ used to describe the Lie algebra $\mathfrak{g}$, the conformal flatness condition is equivalent to the system of algebraic equations

$$
W_{i j k h} R_{i j k h}-\frac{1}{2}\left(g_{i k} \rho_{j h}+g_{j h} \rho_{i k}-g_{i h} \rho_{j k}-g_{j k} \rho_{i h}\right)+\frac{r}{6}\left(g_{i k} g_{j h}-g_{i h} g_{j k}\right)=0,
$$

for all indices $i, j, k, h=1, \ldots, 4$, where $W_{i j k h}$ denote the components of the Weyl tensor with respect to the basis $\left\{e_{i}\right\}$ and $r$ is the scalar curvature.

A conformally flat pseudo-Riemannian manifold of class $\mathscr{A}$ is necessarily Ricci-parallel (hence, locally symmetric), since such spaces have constant scalar curvature and are also in class $\mathscr{B}$, by the Theorem 5.1 of [15]. But the Condition (1.3) is a necessary but not sufficient condition for a pseudoRiemannian manifold of dimension greater or equal to four to be conformally flat. For this reason, we study the conformally flat and Walker examples through the four-dimensional Lorentzian Lie groups of class $\mathscr{B}$. The result is the following proposition.

Proposition 4.1. Among the non-Ricci parallel four-dimensional Einstein-like Lorentzian Lie groups of class $\mathscr{B}$, as classified in Theorem 4.1 up to isometries, the conformally flat examples are:

(i) cases 4), 9), 11), 12) and 13) are always conformally flat.

(ii) case 10) where $A=E-C$ or $(B-F)^{2}+(C+E)^{2}=0$.

(iii) case 14) where $(B-D)^{2}+(A+E-G)^{2}=0$ or $(B+D)^{2}+(A-E)^{2}=0$.

Also, The Walker examples through them are

(a) case 3 ), in this case $X=-\delta e_{1}+e_{3}$ is a left-invariant parallel null line field.

(b) case 6), in this case $X=e_{1}+e_{3}$ is a left-invariant parallel null line field. 
(c) case 8 ), in this case $X=e_{2}+\delta e_{3}$ is a left-invariant parallel null line field.

(d) case 10), in this case $X=e_{3}$ is a left-invariant parallel null line field.

(e) case 14), in this case $X=e_{3}$ is a left-invariant parallel null line field.

Proof. For the Walker structures, we proceed as in the corresponding Theorem 3.2. With regard to conformal flatness, we bring the details of the case 4). The other cases could be treated by similar arguments. Let $G$ be a Lorentzian Lie group of case 4) of the Theorem 4.1. The Lie algebra $\mathfrak{g}$ with respect to the pseudo-orthonoreml basis $\left\{e_{i}\right\}_{i=1}^{4}$ with $e_{3}$ time-like is

$$
\left[e_{1}, e_{4}\right]=(\varepsilon 4 A+3 B) e_{1}-(3 A+\varepsilon 2 B) e_{3},\left[e_{2}, e_{4}\right]=2(\varepsilon A+B) e_{2},\left[e_{3}, e_{4}\right]=A e_{1}+B e_{3},
$$

where $B+\varepsilon A \neq 0, B+\varepsilon 2 A \neq 0$. By using the Koszul formula, one can calculate the Levi-Civita components as

$$
\Lambda_{1}=\left(\begin{array}{cccc}
0 & 0 & 0 & 4 \varepsilon A+3 B \\
0 & 0 & 0 & 0 \\
0 & 0 & 0 & -2 A-\varepsilon B \\
-4 \varepsilon A-3 B & 0 & -2 A-\varepsilon B & 0
\end{array}\right), \Lambda_{2}=\left(\begin{array}{cccc}
0 & 0 & 0 & 0 \\
0 & 0 & 0 & 2 \varepsilon A+2 B \\
0 & 0 & 0 & 0 \\
0-2 \varepsilon A-2 B & 0 & 0 \\
0 & 0 & 0 & 2 A+\varepsilon B \\
0 & 0 & 0 & 0 \\
0 & 0 & 0 & B \\
-2 A \varepsilon B & 0 & B & 0
\end{array}\right), \quad \Lambda_{4}=\left(\begin{array}{cccc}
0 & 0 & 0 & 0 \\
A+\varepsilon B & 0 & 0 & 0 \\
0 & 0 & 0 & 0
\end{array}\right) .
$$

By straight forward but standard calculations we deduce the curvature tensor. If set $R_{i j}=R\left(e_{i}, e_{j}\right)$ have

$$
\begin{aligned}
& R_{12}=\left(\begin{array}{cccc}
0 & -2(4 \varepsilon A+3 B)(\varepsilon A+B) & 0 & 0 \\
2(4 \varepsilon A+3 B)(\varepsilon A+B) & 0 & 2(\varepsilon A+B)(2 A+\varepsilon B) & 0 \\
0 & 2(\varepsilon A+B)(2 A+\varepsilon B) & 0 & 0 \\
0 & 0 & 0 & 0
\end{array}\right), \\
& R_{13}=\left(\begin{array}{cccc}
0 & 0 & 8 \varepsilon A B+4\left(B^{2}+A^{2}\right) & 0 \\
0 & 0 & 0 & 0 \\
8 \varepsilon A B+4\left(B^{2}+A^{2}\right) & 0 & 0 & 0 \\
0 & 0 & 0 & 0
\end{array}\right) \\
& R_{14}=\left(\begin{array}{cccc}
0 & 0 & 0 & -8 A^{2}-14 \varepsilon A B-6 B^{2} \\
0 & 0 & 0 & 0 \\
0 & 0 & 0 & 6 A B+2 \varepsilon\left(B^{2}+2 A^{2}\right) \\
8 A^{2}+14 \varepsilon A B+6 B^{2} & 0 & 6 A B+2 \varepsilon\left(B^{2}+2 A^{2}\right) & 0
\end{array}\right) \text {, } \\
& R_{23}=\left(\begin{array}{cccc}
-2(\varepsilon A+B)(2 A+\varepsilon B) & 2(\varepsilon A+B)(2 A+\varepsilon B) & 0 & 0 \\
0 & 0 & 2 B(\varepsilon A+B) & 0 \\
0 & 0 & 0 & 0 \\
0 & 0 & 0 & 0
\end{array}\right), \\
& R_{24}=\left(\begin{array}{cccc}
0 & 0 & 0 & 0 \\
0 & 0 & 0 & -4(\varepsilon A+B)^{2} \\
0 & 0 & 0 & 0 \\
0 & 4(\varepsilon A+B)^{2} & 0 & 0
\end{array}\right) \\
& R_{34}=\left(\begin{array}{cccc}
0 & 0 & 0 & -6 A B-2 \varepsilon\left(B^{2}+2 A^{2}\right) \\
0 & 0 & 0 & 0 \\
0 & 0 & 0 & -2 \varepsilon A B-2 B^{2} \\
6 A B+2 \varepsilon\left(B^{2}+2 A^{2}\right) & 0-2 \varepsilon A B-2 B^{2} & 0
\end{array}\right) .
\end{aligned}
$$


Now, by using the relation $\rho\left(e_{i}, e_{j}\right)=g\left(R\left(e_{k}, e_{i}\right) e_{j}, e_{k}\right)$ the Ricci tensor is

$$
\rho=\left(\begin{array}{clcc}
-36 \varepsilon A B-4\left(5 A^{2}+4 B^{2}\right) & 0 & \phi & 0 \\
0 & \psi & 0 & 0 \\
\phi & 0 & 12 \varepsilon A B+4\left(A^{2}+2 B^{2}\right) & 0 \\
0 & 0 & 0 & \psi
\end{array}\right),
$$

where $\phi=-12 A B-4 \varepsilon\left(B^{2}+2 A^{2}\right)$ and $\psi=-24 \varepsilon A B-12\left(A^{2}+B^{2}\right)$. Direct calculations using the Equation (4.1) yield that the Weyl conformal tensor vanishes identically in this case. So Lie groups of this case are always conformally flat.

\section{References}

[1] E. Abbena and S. Garbiero, Curvature forms and Einstein-like metrics on Sasakian manifolds, Math. J. Okayama Univ. 34 (1992) 241-248.

[2] E. Abbena, S. Garbiero and L. Vanhecke, Einstein-like metrics on three-dimensional Riemannian homogeneous manifolds, Simon Stevin Quart. J. Pure Appl. Math. 66 (1992) 173-182.

[3] T. Arias-Marco and O. Kowalski, Classification of 4-dimensional homogeneous D'Atri spaces, Czechoslovak Math. J. 58 (2008) 203-239.

[4] L. Bérard-Bérgery, Homogeneous Riemannian spaces of dimension four, Seminar A. Besse, Fourdimensional Riemannian geometry (1985).

[5] E. Boeckx, Einstein-like semi-symmetric spaces, Arch. Math. (Brno) 16 (1997) 789-800.

[6] M. Brozos-Vazquez, E. Garcia-Rio, P. Gilkey, S. Nikcevic and R. Vazquez-Lorenzo, The geometry of Walker manifolds, Synthesis Lect. on Math. and Stat. (Morgan and Claypool Publishers, 2009).

[7] P. Bueken and L. Vanhecke, Three- and four-dimensional Einstein-like manifolds and homogeneity, Geom. Dedicata 75 (1999) 123-136.

[8] G. Calvaruso and L. Vanhecke, Special ball-homogeneous spaces, Z. Anal. Anwendungen 16 (1997) 789-800.

[9] G. Calvaruso, Einstein-like and conformally flat contact metric three-manifolds, Balkan J. Geom. 5(2) (2000) 17-36.

[10] G. Calvaruso, Einstein-like metrics on three-dimensional homogeneous Lorentzian manifolds, Geom. Dedicata 127 (2007) 99-119.

[11] G. Calvaruso, Einstein-like curvature homogeneous Lorentzian three-manifolds, Result. Math. 55 (2009) 295-310.

[12] G. Calvaruso, Einstein-like Lorentz metrics and three-dimensional curvature homogeneity of order one, Canadian Math. Bull. 53 (2010) 412-424.

[13] G. Calvaruso and A. Zaeim, Four-dimensional Lorentzian Lie groups, Differ. Geom. Appl. 31(4) (2013) 496-509.

[14] M. Chaichi, E. García-Río and M. E. Vázquez-Abal, Three-dimensional Lorentz manifolds admitting a parallel null vector field, J. Phys. A: Math. Gen. 38 (2005) 841-850

[15] A. Gray, Einstein-like manifolds whcih are not Einstein, Geom. Dedicata 7 (1978) 259-280.

[16] B. Komrakov Jnr., Einstein-maxwell equation on four-dimensional homogeneous spaces, Lobachevskii J. Math. 8 (2001) 33-165.

[17] J. Milnor, Curvatures of left-invariant metrics on Lie groups, Adv. Math. 21 (1976) 293-329.

[18] S. Rahmani, Métriques de Lorentz sur les groupes de Lie unimodulaires de dimension trois, J. Geom. Phys. 9 (1992) 295-302.

[19] A. G. Walker, On parallel fields of partially null vector spaces, Quart. J. Math. Oxford 20 (1949) 135145 .

[20] A. G. Walker, Canonical form for a Riemannian space with a parallel field of null planes, Quart. J. Math. Oxford 1 (1950), 69-79.

[21] A. Zaeim and A. Haji-Badali, Einstein-like pseudo-Riemannian homogeneous manifolds of dimension four, Mediterr. J. Math. 13(5) (2016) 3455-3468. 